\title{
Just do it: tell the truth
}

\begin{abstract}
Handelt es sich bei der Nachhaltigkeitserklärung einer Firma um unverbindliche Aussagen, die zwar öffentlicher Kritik zugänglich sind aber deren Wahrheitsgehalt gerichtlich nicht gepröft werden kann? Oder sind sie harte Fakten, die einer gerichtlichen Kontrolle standhalten müssen? Eine Klage gegen die Informationspolitik von Nike und die Reaktion des Sportartikelherstellers beleuchten die zentralen Aspekte dieser wichtigen Diskussion.
\end{abstract}

$\mathrm{N}$ Von Ulrich Petschow ike, kampagnengeschädigt wie kaum ein anderes Unternehmen, befindet sich aktuell im Abwehrkampf gegen ein Urteil des obersten Kalifornischen Gerichtshofs. Das Urteil gab Marc Karsky in seiner Klage Recht, Nike hätte gegen kalifornische Gesetze zu falscher Werbung und unfairer Handelspraxis verstoßen.

Als Reaktion ging Nike beim Supreme Court der USA in Revision. Dabei hat sich Nike der Unterstïtzung einer großen Zahl von bekannten Unternehmen, insbesondere auch von Unternehmen der Medienund Werbebranche (auch aus der EU) gesichert. Die von Nike angestrebte Überprüfung des kalifornischen Urteils wurde vom obersten amerikanischen Gericht angenommen, was allein schon die Brisanz des Vorgangs unterstreicht. Die durchschnittliche Ablehnungsquote von Anträgen zur Überprüfung von Urteilen liegt bei 99 Prozent. Eine Entscheidung wird für Ende Juni diesen Jahres erwartet.

Zur Verhandlung steht nicht weniger als das first amendment der amerikanischen Verfassung: die freie Rede. Der Hintergrund der Auseinandersetzung: Der Sportartikelhersteller Nike lässt seine Produkte durch Subkontraktoren in 736 Betriebsstätten mit etwa 500.000 Beschäftigten in 51 Ländern weltweit fertigen. Der größte Teil der Produktion findet in China, Vietnam und Indonesien statt (1).

Nike hat mit allen Zulieferern Vereinbarungen zu den Produktionsbedingungen für Nike-Artikel, so genannte "Memoranda of Understanding", getroffen. In den Vereinbarungen verpflichten sich die Zulieferer, die jeweiligen nationalen Gesetze bezüglich Mindestlöhnen, Kinderarbeit, Arbeitsbedingungen etc. einzuhalten und die Einhaltung zu dokumentieren. Die Einhaltung dieser Verpflichtungen wurden 1997 durch externe Auditoren unter der Leitung des ehemaligen UN-Botschafters der USA
Young bestätigt. Nachdem Young und sein Team zwölf Zulieferer inspiziert hatten, stellten sie Nike in der Summe ein gutes Zeugnis aus.

Fast zeitgleich dokumentierten eine Reihe anderer Untersuchungen massive Verletzungen der von den Subunternehmern eingegangenen Selbstverpflichtungsregeln und stellten die Untersuchungen von Young massiv in Frage. Eine weltweites, Nikekritisches Medienecho ließ nicht lange auf sich warten.

Mit den Untersuchungsergebnissen von Young im Rücken ging nun Nike in die Offensive. Die NikeFabriken in Entwicklungsändern kämen den einzelnen Ländern zu Gute und die Behauptungen aller Young widersprechenden Untersuchungen seien falsch, waren die zentralen Botschaften der entsprechenden PR- und Werbekampagne - Werbeund Marketingausgaben machten 1997 über zehn Prozent des Konzernumsatzes aus.

\section{Das kalifornische Urteil}

In seiner Klage legte Karsky dar, dass Nike in seiner Kampagne sechs Falschdarstellungen gemacht habe, deren Ziel es sei den Produktabsatz aufrecht zu erhalten und zu steigern. Damit habe das Unternehmen gegen die kalifornischen Gesetze zu falschen Werbeaussagen und unfairer Handelspraxis verstoßen. Nike verteidigte sich gegen Karkys Vorwüfe mit dem Hinweis auf das Recht zur freien Rede.

Die kalifornischen Richter mussten im Fall Karsky gegen Nike entscheiden, ob Unternehmen die gleichen Rechte wie Individuen haben, wenn sie öffentliche Aussagen machen. Die dem Fall zu Grunde liegende rechtliche Frage ist, ob die Aussagen der Firma Nike im Rahmen ihrer Öffentlichkeitsarbeit normal speech - und damit durch das Recht zur Meinungsfreiheit, egal ob richtig oder falsch, vor Klagen geschützt - sind. Der Supreme Court von Kalifornien entschied aber mit einer knappen Vier zu Drei-Mehrheit, dass die öffentliche Verteidigung von Nike Werbung (commercial speech) darstellt, weil die Aussagen von Nike auf die Konsumenten ihrer Produkte zielten.

Die drei Richter, die gegen diese Klassifizierung von Nikes Aussagen gestimmt hatten, begründeten ihre Entscheidung mit dem Hinweis auf die grundsätzliche Unmöglichkeit der eindeutigen Klassifizierung von Aussagen. So wie die Grenzen zwischen Werbung, Kunst und Politik aufweichten, so die Richter, sei auch die in früheren Urteilen unterstellte Trennung zwischen kommerziell und künstlerisch-politisch in Frage gestellt. Wenn die Möglichkeit der Unterscheidung zwischen Werbung, politischer Aussage und letztlich "Wahrheit" zunehmend eingeschränkt sei, solle auch Werbung dem Schutz der freien Meinungsäußerung unterliegen.

\section{Die Folgen}

Die europäischen Unterstïtzer von Nike glauben, dass das kalifornische Urteil sich auch schädlich auf die Politik in der EU auswirken könne. Sie befürchten, dass die Unternehmenstransparenz bei sozialen, ethischen und Umweltthemen entmutigt wird, die die EU zu einem zentralen Bestandteil ihrer Unterstïtzung für nachhaltiges Investment gemacht hat.

Nike reagierte, indem es seine bisherigen Publikationen etwa des Social Responsibility Reports zurückhält und zugleich die Kommunikation mit der Außenwelt bei problematischen Themen einschränkt, um weiteren Klagen vorzubeugen.

Der Fall verdeutlicht die Probleme von freiwilligen Selbstverpflichtungen: die Aussagen sind vielfach nicht zu kontrollieren, kritische Berichte werden nicht widerlegt, sondern eigenen Auditoren zitiert und deren Berichte als Bestandteil der Marketingund Reputationsmaßnahmen verbreitet. Mithin steht nicht allein die Frage der commercial speech sondern der "Wahrheits"gehalt freiwilliger Selbstverpflichtungen der Unternehmen zum Schutz der Unternehmensreputation auf dem Prüfstand.

\section{Anmerkungen}

(1) Vgl. für weitere Informationen zum Fall und weitergehende Links www.ReclaimDemocracy.org sowie www.nike.org

\section{Der Autor}

Ulrich Petschow ist Leiter des Forschungsfelds Umweltökonomie und -politik des Instituts für ökologische Wirtschaftsforschung (IÖW). Kontakt: IÖW, Potsdamer Str. 105, 10785 Berlin. Tel. 030-884594-0, E-Mail: ulrich.petschow@ioew.de 
(c) 20I0 Authors; licensee IÖW and oekom verlag. This is an article distributed under the terms of the Creative Commons Attribution Non-Commercial No Derivates License (http://creativecommons.org/licenses/by-nc-nd/3.o/), which permits unrestricted use, distribution, and reproduction in any medium, provided the original work is properly cited. 